\title{
Hydro overview
}

\author{
Jean-Yves Ollitrault ${ }^{\mathrm{a}}$, Fernando G. Gardim ${ }^{\mathrm{b}}$
}

${ }^{a}$ CNRS, URA2306, IPhT, Institut de physique théorique de Saclay, F-91191 Gif-sur-Yvette, France

${ }^{b}$ Instituto de Física, Universidade de São Paulo, C.P. 66318, 05315-970, São Paulo-SP, Brazil

\begin{abstract}
We review recent progress in applying relativistic hydrodynamics to the modeling of heavy-ion collisions at RHIC and LHC, with emphasis on anisotropic flow and flow fluctuations.
\end{abstract}

\section{Introduction}

Relativistic hydrodynamics is the only first-principles approach [1] to the out-of-equilibrium dynamics of the strongly-coupled quark-gluon plasma formed in heavy-ion collisions. It is a macroscopic description in which the plasma is modeled as a continuous lump of fluid expanding into the vacuum. It plays an important role in understanding the soft sector of nucleus-nucleus collisions at RHIC and LHC.

The goal of relativistic hydrodynamics is to describe the bulk of particle production. A hydrodynamic calculation uses as input a model for initial conditions - typically, for the initial density profile in a collision. The only input needed for hydrodynamics is the initial value of the energy-momentum tensor: all other microscopic details of the initial state are washed out by local thermalization, which is implicitly assumed by the hydrodynamic description. One then solves for the fluid expansion using equations of ideal or viscous relativistic hydrodynamics. Eventually, the fluid is transformed into independent hadrons 1

The fluid is continuous: single-particle distributions from the fluid can be computed (as a function of transverse momentum $p_{t}$, pseudorapidity $\eta$, azimuthal angle $\phi$ ) with unlimited accuracy for a given initial condition, unlike in an actual experiment. As we shall see later, this peculiar feature of the fluid description is an important one. Many groups implement hadronization using a Monte-Carlo generator, thus mimicking the experimental situation where at most a few thousand particles are observed per even ${ }^{2}$ This amounts to picking randomly one element out of a thermal ensemble. The fluid is the thermal ensemble.

In 2010, it was shown that observed azimuthal correlations between particles separated by a gap in pseudorapidity (usually referred to as "long-range" correlations) are compatible with this hydrodynamic picture [2, 3], thus providing a unique signature of collective behavior. In these proceedings, we review the status of this "flow hypothesis" in light of recent measurements. We then list open issues, and recent works since the last Quark Matter conference.

\footnotetext{
${ }^{1}$ Depending on the implementation, further hadronic decays and/or rescatterings may occur after hadronization.

${ }^{2}$ This is the most natural way of implementing a "hadronic afterburner", i.e., further rescatterings after hadronization.
} 

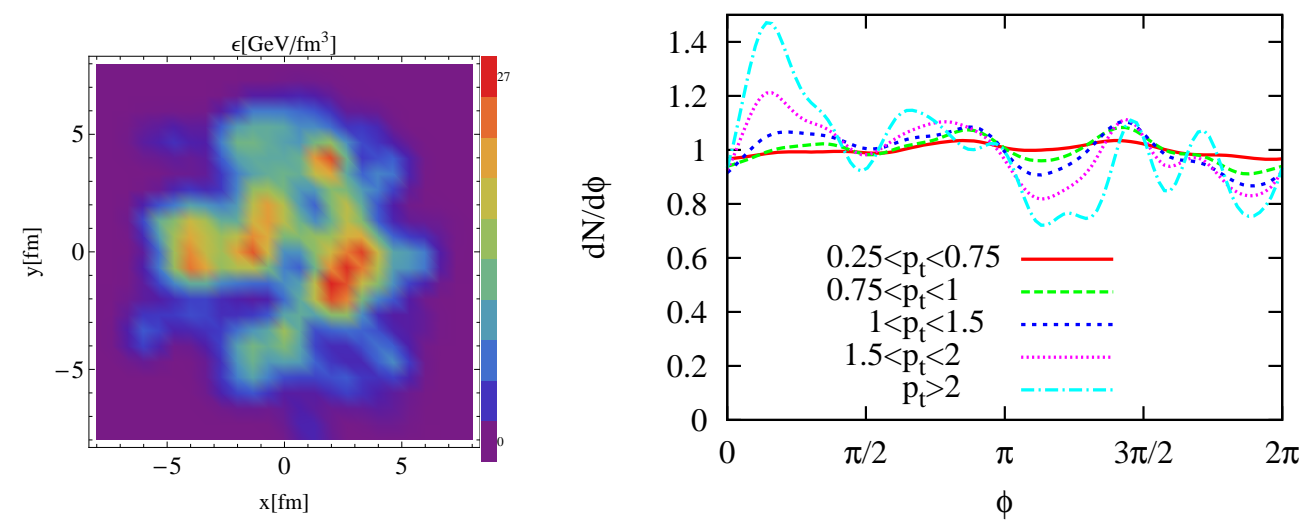

Figure 1: (Color online) Left: initial energy density in the transverse plane at $z=0$ in a random central $(b=0) \mathrm{Pb}-\mathrm{Pb}$ collision at $2.76 \mathrm{TeV}$ simulated by Nexus. Right: azimuthal distribution of outgoing particles after evolving this initial distribution through ideal hydrodynamics, in several bins of transverse momentum $p_{t}$ (in units of $\mathrm{GeV} / c$ ).

\section{A close look at a hydro event}

Quantum fluctuations in the wavefunctions of colliding nuclei result in an initial profile which is not smooth and fluctuates from event to event [4]. In order to understand the physics associated with these initial fluctuations, it is instructive to look at a particular event in some detail. We simulate a central $(b=0) \mathrm{Au}-\mathrm{Au}$ collision using the event generator NeXus [5], which gives the initial density profile shown in Fig. 1](left). These initial conditions are then evolved through ideal hydrodynamics [6]. As explained above, thermalization thus transforms a single event into a thermal ensemble. The term "hydro event" usually refers to this thermal ensemble. We transform the fluid into hadrons using a Monte-Carlo generator. This hadronization is repeated a few thousand times so that we can reliably calculate ensemble-averaged quantities for the single hydro event displayed in Fig. 11 (left).

A central collision between spherical nuclei is azimuthally symmetric, except for quantum fluctuations. One clearly sees in Fig. 1 (left) that fluctuations break rotational symmetry in the transverse plane. Fig. 1 (right) displays the azimuthal distribution of charged particles in the pseudorapidity interval $|\eta|<1$ for various bins in $p_{t}$. Anisotropies are at the $\%$ level at low $p_{t}$ and become stronger and stronger as $p_{t}$ increases.

The azimuthal distribution can be expressed as a Fourier series:

$$
\frac{2 \pi}{N} \frac{d N}{d \phi}=\sum_{n=-\infty}^{\infty} V_{n}\left(p_{t}, \eta\right) e^{-i n \phi},
$$

where $V_{n}=\left\{e^{i n \phi}\right\}$ is the $n$th (complex) Fourier coefficient, and curly brackets indicate an average over the probability density in a single event. Writing $V_{n}=v_{n} e^{i n \Psi_{n}}$, where $v_{n}$ is the (real) anisotropic flow coefficient and $\Psi_{n}$ the corresponding phase, and using $V_{-n}=V_{n}^{*}$ (where the superscript * denotes the complex conjugate), this can be rewritten as

$$
\frac{2 \pi}{N} \frac{d N}{d \phi}=1+2 \sum_{n=1}^{\infty} v_{n}\left(p_{t}, \eta\right) \cos n\left(\phi-\Psi_{n}\left(p_{t}, \eta\right)\right) .
$$



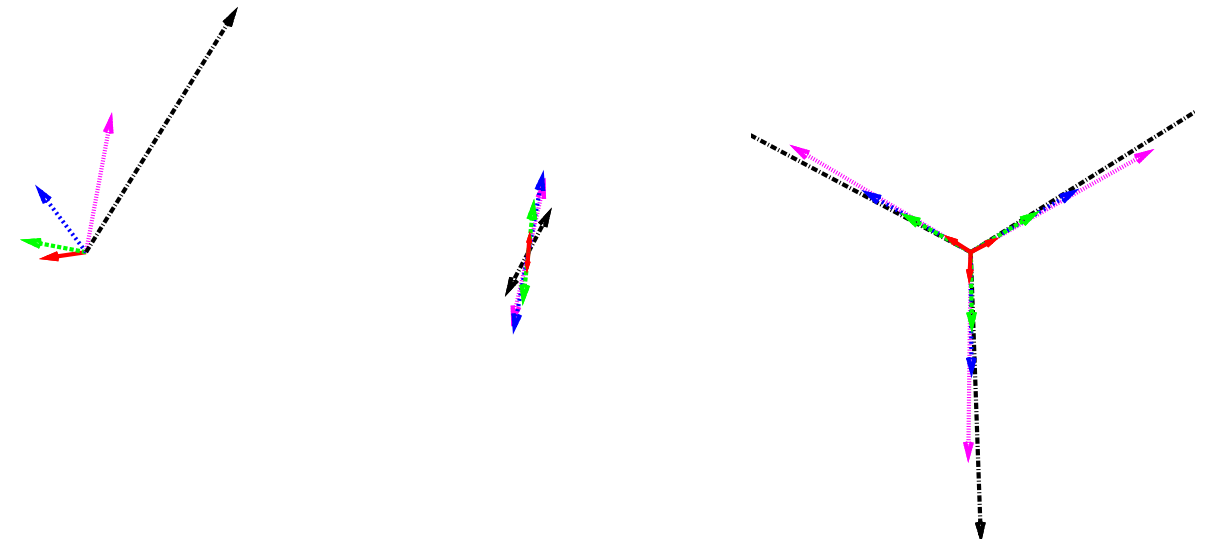

Figure 2: (Color online) Arrows representing the magnitude and direction of the first Fourier coefficients of the curves in Fig. 1 (right). The length of the arrow is $v_{n}$ and its orientation is $\Psi_{n}$. From left to right: $n=1$ (directed flow), $n=2$ (elliptic flow), $n=3$ (triangular flow).

Note that, for this form to describe an arbitrary distribution, both $v_{n}$ and $\Psi_{n}$ may depend on transverse momentum $p_{t}$ and pseudorapidity $\eta$. Most models of initial conditions predict fluctuations in the form of longitudinally-extended flux tubes [7]. Therefore, one expects $\Psi_{n}$ and $v_{n}$ to depend little on rapidity. Although this rapidity dependence is worth investigating [8, 9, 10], we focus here on the stronger $p_{t}$ dependence.

Note that event-by-event anisotropic flow is thus well defined in hydrodynamics only, because thermalization transforms a single event into a thermal ensemble. In an actual experiment, the relative statistical error is typically $50 \%$ for $v_{2}$ (in a mid-central $\mathrm{Pb}-\mathrm{Pb}$ collision analyzed by CMS or ATLAS), and $100 \%$ for $v_{3}$. Therefore the event-by-event $v_{n}$, as defined above from the single-particle distribution, cannot be measured experimentally.

Fig. 2 displays the magnitude $\left(v_{n}\right)$ and direction $\left(\Psi_{n}\right)$ of the first three harmonics obtained by a Fourier decomposition of the distributions in Fig. 1. Note that $\Psi_{n}$ is defined modulo $2 \pi / n$, and is therefore represented using $n$ arrows pointing into the corresponding directions. As noted before, azimuthal anisotropies generally increase with $p_{t}$, hence $v_{n}$ increases with $p_{t}$. The directions of elliptic flow $\left(\Psi_{2}\right)$ and triangular flow $\left(\Psi_{3}\right)$ depend slighly on $p_{t}^{3}$ The $p_{t}$ dependence is much stronger for $\Psi_{1}$. This reflects the fact that the net transverse momentum of the central rapidity slice is expected to be close to 0 , hence the integral of $p_{t} V_{1}\left(p_{t}\right)=p_{t} v_{1}\left(p_{t}\right) e^{i \Psi_{1}\left(p_{t}\right)}$ over all particles should be 0 . One therefore typically expect that $\Psi_{1}$ rotates by $\pi$ as transverse momentum goes from 0 to $\propto 4$

\section{Flow in data: a close look at 2-particle correlations}

As explained above, event-by-event anisotropic flow is well defined in hydrodynamics only. The number of particles per event in an actual experiment is too small to measure $V_{n}\left(p_{t}, \eta\right)$, or

\footnotetext{
${ }^{3}$ The particular event that we randomly picked for this illustration turns out to have a rather small $v_{2}$ and large $v_{3}$.

${ }^{4}$ The hydrodynamic code (NeXSPheRIO) that we use has a small residual net transverse momentum due to the choice of initial conditions, as noted in a previous study of directed flow [11]. This effect has not been corrected here.
} 
even the integrated $v_{n}$. Anisotropic flow can only be measured through event-averaged azimuthal correlations between particles. The simplest azimuthal correlation is the pair correlation. One forms all possible pairs of particles and then performs a harmonic decomposition of the distribution of the relative angle:

$$
V_{n \Delta}\left(p_{t}^{a}, p_{t}^{b}\right) \equiv\left\langle\cos \left(n\left(\phi_{a}-\phi_{b}\right)\right)\right\rangle=\left\langle e^{i n\left(\phi_{a}-\phi_{b}\right)}\right\rangle .
$$

This quantity can be measured as a function of the transverse momenta of both particles, labeled as $a$ and $b$, thus yielding a correlation matrix. This correlation matrix has recently been measured by LHC experiments [12, 13, 14]. It is symmetric by construction ${ }^{5}$

We now evaluate this quantity in hydrodynamics and show that it is can be expressed simply in terms of anisotropic flow. In a single hydro event, particles are emitted independently. Therefore

$$
\left\{e^{i n\left(\phi^{a}-\phi^{b}\right)}\right\}=\left\{e^{i n \phi^{a}}\right\}\left\{e^{-i n \phi^{b}}\right\}=V_{n}^{a} V_{n}^{b *}=v_{n}^{a} v_{n}^{b} e^{i n\left(\Psi_{n}^{a}-\Psi_{n}^{b}\right)},
$$

where $\{\cdots\}$ denotes an average over a single hydro event, The first equality in Eq. (4) expresses mathematically that particles are independent: this implies that the two-particle correlation, when written in complex form, factorizes in a single hydro event.

The experimental quantity, Eq. (3), is then obtained by averaging over a larger number of hydro events:

$$
V_{n \Delta}\left(p_{t}^{a}, p_{t}^{b}\right)=\left\langle V_{n}^{a} V_{n}^{b *}\right\rangle=\left\langle v_{n}^{a} v_{n}^{b} e^{i n\left(\Psi_{n}^{a}-\Psi_{n}^{b}\right)}\right\rangle
$$

Due to parity symmetry, only the real part remains after this average, hence the cosine in Eq. (3).

From this relation alone, one can make the following general statements about the eventaveraged correlation matrix when flow is dominant: the diagonal elements must be positive, and the off-diagonal elements must satisfy a Cauchy-Schwarz inequality [15],

$$
\begin{aligned}
V_{n \Delta}\left(p_{t}^{a}, p_{t}^{a}\right) & \geq 0, \\
\left|V_{n \Delta}\left(p_{t}^{a}, p_{t}^{b}\right)\right|^{2} & \leq V_{n \Delta}\left(p_{t}^{a}, p_{t}^{a}\right) V_{n \Delta}\left(p_{t}^{b}, p_{t}^{b}\right) .
\end{aligned}
$$

It is often stated that flow implies factorization. As shown above, factorization holds for a single hydro event. It implies that the second inequality in (6) is saturated, i.e., equality is achieved. After averaging over events, one does not in general expect factorization to hold, therefore the 2 nd inequality is generally a strict one for $p_{t}^{a} \neq p_{t}^{b}$. Any violation of $(6)$ is an unambiguous indication of the presence of non-flow correlations.

Inequalities (6) can be directly tested on experimental data. We use ALICE data [12] for $0-10 \%$ central $\mathrm{Pb}-\mathrm{Pb}$ collisions. For $n=2$ (Fig. 3, middle), all diagonal elements are positive, and the Cauchy-Schwarz is verified except for two matrix elements, where the violation is compatible with a statistical fluctuation (the deviation is barely above $1 \sigma$ ). The ALICE collaboration concluded from their analysis that the correlation matrix factorizes approximately for trigger particles below $4 \mathrm{GeV} / c$. However, deviations from factorization are clearly seen at much lower transverse momentum. It is interesting to note that they are everywhere compatible with the general inequalities predicted by hydrodynamics. If hydrodynamics is a valid approach, such deviations are a direct evidence of event-by-event fluctuations.

For $n=3$ (Fig. 3 , right), diagonal elements are negative above $5 \mathrm{GeV}$, thus violating the first inequality (6) and providing evidence for breakdown of the independent-particle hypothesis

\footnotetext{
${ }^{5}$ ATLAS [14] uses a different binning in $p_{t}$ for the two particles in the pair, thus breaking the symmetry.
} 

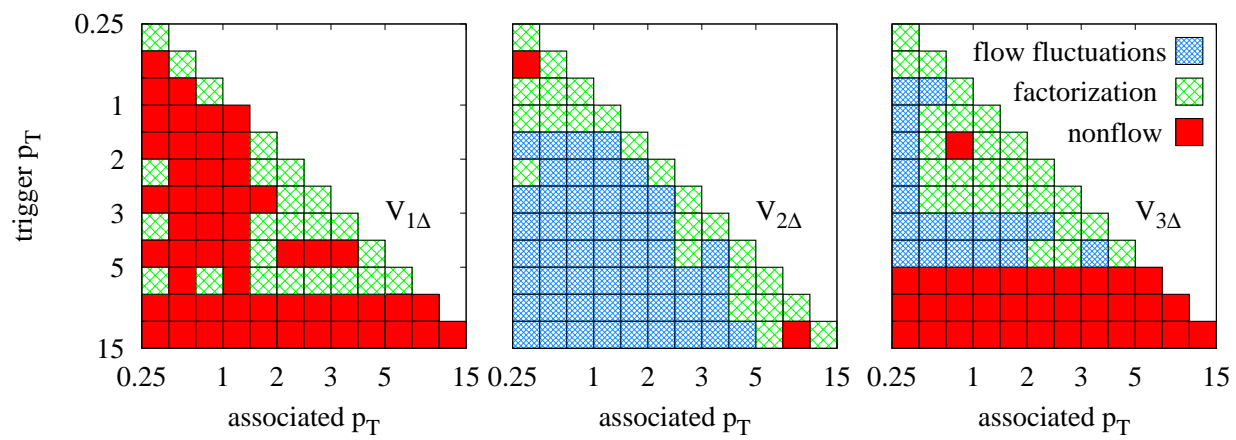

Figure 3: (Color online) Test of inequalities $\sqrt{6}$ for the correlation matrix $V_{n \Delta}\left(p_{t}^{a}, p_{t}^{b}\right)$ measured by ALICE [12] for the $10 \%$ most central $\mathrm{Pb}-\mathrm{Pb}$ collisions at $2.76 \mathrm{TeV}$. Green: the second inequality is an equality within errors. Blue: strict inequality (flow fluctuations). Red: inequalities violated (correlations cannot be explained by hydrodynamics). From left to right: $n=1,2,3$. Only statistical errors were taken into account. The boundaries of the $p_{t}$ bins are $0.25,0.5,0.75,1$, $1.5,2,2.5,3,4,5,6,8,15$ (in $\mathrm{GeV} / c$ ). We use the standard terminology and denote by trigger (associated) the particle with the higher (lower) $p_{t}$, although this distinction is irrelevant here.

underlying the hydrodynamic picture. The observed effect can be qualitatively explained by correlations from jets: the trigger and associated particles are separated by a pseudorapidity gap and cannot belong to the same jet. But they can belong to the same pair of back-to-back jets, in which case their azimuthal angles are typically separated by $\Delta \phi \simeq \pi$, thus giving a negative contribution to diagonal elements for odd $n$. Below $5 \mathrm{GeV}$, all matrix elements are compatible with inequalities (6), except for one point (which is again compatible with a statistical fluctuation). As observed for $n=2$, factorization is broken even at low $p_{t}$.

Finally, for $n=1$ (Fig. 3. left), the inequalities (6) are massively violated, both at low and high $p_{t}$, thus indicating that nonflow effects are important. One of these nonflow effects is the correlation induced by global momentum conservation, which only contributes to the first Fourier harmonic. After correcting for this effect, data are dominated by collective flow [14, 16].

Comparisons between hydro and data so far only address the "single particle $v_{n}\left(p_{t}\right)$ ", which is inferred from the correlation between a single particle with momentum $p_{t}$ and all the particles in a reference detector. Such measurements amount to averaging the correlation matrix, Fig. 3, over a line or a column. As we have shown above, the detailed structure of the matrix contains much more information; in particular, it provides direct insight into event-by-event fluctuations. The correlation matrix defined by Eq. (5) can be directly evaluated in event-by-event hydrodynamics. Future event-by-event hydrodynamics should address the full structure of two-particle correlations, which can be directly compared with experimental data.

For the sake of brevity, we have only discussed the simplest correlations, i.e., pair correlations. Much additional information is contained in higher-order correlations. Higher-order cumulants give direct information on the magnitude of event-by-event flow fluctuations [17]. A much wider range of possibilities is opened up by mixed correlations between event planes, which have recently been measured at the LHC [18, 19] and can be directly evaluated in eventby-event hydrodynamic calculations [20]. Such higher-order correlations are likely to play an important part in the near future of hydrodynamic calculations. 


\section{Recent progress and open issues}

It has long been recognized [21] that the dominant source of uncertainty in comparisons between hydro and experimental data lies in the modeling of initial conditions. The modeling of event-by-event fluctuations introduces further uncertainty and has triggered much recent activity [22, 23, 24]. From the point of view of hydrodynamics, it is important to understand the hydrodynamic response to a given initial condition: for instance, to what extent elliptic flow represents the response to the initial eccentricity of the interaction region. This hydrodynamic response has been studied quantitatively [25] and the importance of nonlinear terms has been pointed out [26].

As for the hydrodynamic expansion itself, the dominant source of uncertainty is the value of transport coefficients [27]. Bulk viscosity was recently shown to have a small effect on integrated flow observables [28, 29]. Shear viscosity, on the other hand, is known to reduce anisotropic flow: the higher the harmonic, the stronger the effect [30]. But higher harmonics also have a larger uncertainty from initial conditions: presently, RHIC data are equally well reproduced with a minimal shear viscosity [31] or with ideal hydrodynamics [32], depending on how initial conditions are modeled. Studying ultra-central collisions at the LHC may help reducing the uncertainty on the shear viscosity [33].

The last stage of the hydrodynamic evolution is the hadronic phase. It is not clear at present whether hadronic interactions are strong enough for hydrodynamics to be a valid description of this phase. There seems to be a consensus that some hadronic interactions are needed in order to match spectra of identified hadrons, but that ideal hydrodynamics fails. A transport calculation (hadronic afterburner) reproduces data quite well [34]. Alternatively, one can use hydrodynamics with a non-vanishing bulk viscosity [35].

Table 1 is a list of papers published since the last Quark Matter conference and containing numerical hydrodynamic calculations applied to ultrarelativistic nucleus-nucleus collisions. The list is by no means exhaustive. In particular, it does not include theoretical developments: Progress has been made in understanding the relationship between transport theory and hydrodynamics [50], which is relevant to our understanding of the hadronic phase. Another important development is the relativistic theory of hydrodynamical fluctuations [51]. The correlations resulting from such intrinsic fluctuations [42, 52], which are typically thought of as "nonflow" correlations, could thus eventually be studied within the framework of hydrodynamics [53].

\section{Perspectives}

Hydrodynamics has been the state-of-the-art approach to the soft sector of nucleus-nucleus collisions for at least a decade. It was first used to explain the large value of elliptic flow at RHIC. Other azimuthal correlations, such as the "soft ridge" [54], were largely thought to be "nonflow" effects, and it took a few years to recognize [2] that they were also naturally described by hydrodynamics. In these proceedings, we have pointed out for the first time that the detailed structure of azimuthal correlations [12] might be explained by hydrodynamics alone below $5 \mathrm{GeV}$. Hydrodynamics encompasses a wider and wider range of phenomena.

The field of nucleus-nucleus collisions is characterized by a strong interplay between theory and experiment. Most experimental measurements of anisotropic flow are biased by the hydrodynamic picture where flow is a single-particle observable. Elliptic flow is often presented as a 


\begin{tabular}{|c|c|c|c|c|c|}
\hline Author & Ref. & initial fluctuations & $3+1 d$ & viscous & afterburner \\
\hline Dusling & [28] & & & $\checkmark$ & \\
\hline Schenke & [31] & $\checkmark$ & $\checkmark$ & $\checkmark$ & \\
\hline Derradi de Souza & [17] & $\checkmark$ & $\checkmark$ & & \\
\hline Yan YL & [36] & & $\checkmark$ & & $\checkmark$ \\
\hline Chaudhuri & [37] & $\checkmark$ & & $\checkmark$ & \\
\hline Petersen & [38] & $\checkmark$ & $\checkmark$ & & $\checkmark$ \\
\hline Vredevoogd & [39] & & $\checkmark$ & $\checkmark$ & \\
\hline Shen C & [40] & & & $\checkmark$ & \\
\hline Gardim & [32] & $\checkmark$ & $\checkmark$ & & \\
\hline Retinskaya & [16] & & & $\checkmark$ & \\
\hline Ryblewski & [41] & & $\checkmark$ & & \\
\hline Bozek & [42] & $\checkmark$ & $\checkmark$ & $\checkmark$ & \\
\hline Nonaka & [43] & $\checkmark$ & $\checkmark$ & $\checkmark$ & \\
\hline Karpenko & [44] & & $\checkmark$ & & $\checkmark$ \\
\hline Hirano & [45] & $\checkmark$ & $\checkmark$ & & $\checkmark$ \\
\hline Pang LG & [10] & $\checkmark$ & $\checkmark$ & & \\
\hline Teaney & [26] & & & $\checkmark$ & \\
\hline Song H & [46] & & & $\checkmark$ & $\checkmark$ \\
\hline Holopainen & 47] & $\checkmark$ & & & \\
\hline Soltz & [48] & & & $\checkmark$ & $\checkmark$ \\
\hline Qiu Z & [20] & $\checkmark$ & & $\checkmark$ & \\
\hline Luzum & [33] & & & $\checkmark$ & \\
\hline Ryu & [49] & $\checkmark$ & $\checkmark$ & $\checkmark$ & $\checkmark$ \\
\hline
\end{tabular}

Table 1: Recent works (since the Quark Matter 2011 conference, listed in chronological order) containing numerical hydrodynamic calculations applied to heavy-ion collisions. We indicate by check marks whether or not calculations involve event-by-event fluctuations of the initial state; whether they are three dimensional or two-dimensional (with Bjorken longitudinal expansion); whether they are ideal or viscous; and finally, whether or not collisions are implemented in the hadronic phase (hadronic afterburner).

"single-particle" observable, even though all measurements are inferred from azimuthal correlations. Triangular flow was actually discovered [2] through a thorough analysis of these correlations. We advocate that experiments measure quantities which are simple and unambiguous. The pair correlation (3) is a good example of such a simple - yet non-trivial- measurement. In turn, theory should directly address what is measured, i.e., correlations. This will allow for a more fruitful interplay between theory and experiment.

Hydrodynamics applied to ultrarelativistic heavy-ion collisions is an active and lively field of research, and the recent measurements open up exciting new perspectives. The structure of pair correlations will be investigated as a function of transverse momentum and rapidity. The recently-measured correlations between event planes [18, 19] will also further constrain models and tighten our knowledge of the initial state.

\section{Acknowledgments}

We thank Frédérique Grassi and Matthew Luzum for discussions. JYO is supported by the European Research Council under the Advanced Investigator Grant ERC-AD-267258. 


\section{References}

[1] R. Baier et al., JHEP 0804 (2008) 100, 0712.2451.

[2] B. Alver and G. Roland, Phys.Rev. C81 (2010) 054905, 1003.0194.

[3] M. Luzum, Phys.Lett. B696 (2011) 499, 1011.5773.

[4] PHOBOS Collaboration, B. Alver et al., Phys.Rev.Lett. 98 (2007) 242302, nucl-ex/0610037.

[5] H. Drescher et al., Phys.Rept. 350 (2001) 93, hep-ph/0007198.

[6] Y. Hama, T. Kodama and J. Socolowski, O., Braz.J.Phys. 35 (2005) 24, hep-ph/0407264.

[7] A. Dumitru et al., Nucl.Phys. A810 (2008) 91, 0804.3858.

[8] P. Bozek, W. Broniowski and J. Moreira, Phys.Rev. C83 (2011) 034911, 1011.3354.

[9] S. Florchinger and U.A. Wiedemann, JHEP 1111 (2011) 100, 1108.5535.

[10] L. Pang, Q. Wang and X.N. Wang, Phys.Rev. C86 (2012) 024911, 1205.5019.

[11] F.G. Gardim et al., Phys.Rev. C83 (2011) 064901, 1103.4605.

[12] ALICE Collaboration, K. Aamodt et al., Phys.Lett. B708 (2012) 249, 1109.2501.

[13] CMS Collaboration, S. Chatrchyan et al., Eur.Phys.J. C72 (2012) 2012, 1201.3158

[14] ATLAS Collaboration, G. Aad et al., Phys.Rev. C86 (2012) 014907, 1203.3087.

[15] F.G. Gardim et al., (2012), in preparation.

[16] E. Retinskaya, M. Luzum and J.Y. Ollitrault, Phys.Rev.Lett. 108 (2012) 252302, 1203.0931.

[17] R. Derradi de Souza et al., Phys.Rev. C85 (2012) 054909, 1110.5698.

[18] ATLAS Collaboration, J. Jia, (2012), 1208.1427.

[19] ALICE Collaboration, A. Bilandzic, (2012), 1210.6222.

[20] Z. Qiu and U.W. Heinz, Phys.Lett. B717 (2012) 261, 1208.1200.

[21] M. Luzum and P. Romatschke, Phys.Rev. C78 (2008) 034915, 0804.4015.

[22] M. Alvioli et al., Phys.Rev. C85 (2012) 034902, 1112.5306.

[23] A. Dumitru and Y. Nara, Phys.Rev. C85 (2012) 034907, 1201.6382.

[24] B. Schenke, P. Tribedy and R. Venugopalan, Phys.Rev. C86 (2012) 034908, 1206.6805.

[25] F.G. Gardim et al., Phys.Rev. C85 (2012) 024908, 1111.6538.

[26] D. Teaney and L. Yan, (2012), 1206.1905.

[27] H. Song, (2012), 1210.5778.

[28] K. Dusling and T. Schafer, Phys.Rev. C85 (2012) 044909, 1109.5181.

[29] T. Schaefer and K. Dusling, (2012), 1210.4113.

[30] B.H. Alver et al., Phys.Rev. C82 (2010) 034913, 1007.5469.

[31] B. Schenke, S. Jeon and C. Gale, Phys.Rev. C85 (2012) 024901, 1109.6289.

[32] F.G. Gardim et al., (2012), 1203.2882.

[33] M. Luzum and J.Y. Ollitrault, (2012), 1210.6010.

[34] H. Song, S.A. Bass and U. Heinz, Phys.Rev. C83 (2011) 054912, 1103.2380.

[35] P. Bozek and I. Wyskiel-Piekarska, Phys.Rev. C85 (2012) 064915, 1203.6513.

[36] Y.L. Yan et al., (2011), 1110.6704.

[37] A. Chaudhuri, Phys.Lett. B713 (2012) 91, 1112.1166.

[38] H. Petersen, R. La Placa and S.A. Bass, J.Phys. G39 (2012) 055102, 1201.1881.

[39] J. Vredevoogd and S. Pratt, Phys.Rev. C85 (2012) 044908, 1202.1509.

[40] C. Shen and U. Heinz, Phys.Rev. C85 (2012) 054902, 1202.6620.

[41] R. Ryblewski and W. Florkowski, Phys.Rev. C85 (2012) 064901, 1204.2624.

[42] P. Bozek and W. Broniowski, Phys.Rev.Lett. 109 (2012) 062301, 1204.3580.

[43] C. Nonaka and M. Asakawa, (2012), 1204.4795.

[44] I. Karpenko, Y. Sinyukov and K. Werner, (2012), 1204.5351.

[45] T. Hirano et al., (2012), 1204.5814.

[46] H. Song, (2012), 1207.2396.

[47] H. Holopainen and P. Huovinen, (2012), 1207.7331.

[48] R. Soltz et al., (2012), 1208.0897.

[49] S. Ryu et al., (2012), 1210.4588.

[50] G. Denicol et al., Phys.Rev. D85 (2012) 114047, 1202.4551

[51] J. Kapusta, B. Muller and M. Stephanov, Phys.Rev. C85 (2012) 054906, 1112.6405.

[52] S. Gavin and G. Moschelli, Phys.Rev. C86 (2012) 034902, 1205.1218.

[53] T. Springer and M. Stephanov, (2012), 1210.5179.

[54] PHOBOS Collaboration, B. Alver et al., Phys.Rev. C81 (2010) 024904, 0812.1172. 